\title{
Autologous adipose tissue-derived mesenchymal stem cells are involved in rat liver regeneration following repeat partial hepatectomy
}

\author{
TAO LIU ${ }^{1,2}$, HONG MU ${ }^{1}$, ZHONGYANG SHEN ${ }^{2}$, ZHUOLUN SONG $^{2}$, XIAOBO CHEN ${ }^{3}$ and YULIANG WANG ${ }^{1,2}$ \\ Departments of ${ }^{1}$ Clinical Laboratory Medicine and ${ }^{2}$ Transplantation Surgery, Tianjin First Central Hospital, \\ Key Laboratory for Critical Care Medicine of the Ministry of Health, Tianjin 300192; \\ ${ }^{3}$ Union Stem and Gene Engineering Co., Ltd., Tianjin 300384, P.R. China
}

Received January 14, 2015; Accepted October 26, 2015

DOI: $10.3892 / \mathrm{mmr} .2016 .4768$

\begin{abstract}
Adipose tissue-derived mesenchymal stem cells (ADSCs) have been considered to be attractive and readily available adult mesenchymal stem cells, and they are becoming increasingly popular for use in regenerative cell therapy, as they are readily accessible through minimally invasive techniques. The present study investigated whether autologous ADSC transplantation promoted liver regeneration following a repeat partial hepatectomy in rats. The rats were divided into three groups as follows: $70 \%$ partial hepatectomy $(\mathrm{PH})$ group; repeat $\mathrm{PH}(\mathrm{R}-\mathrm{PH})$ group and R-PH/ADSC group, subjected to R-PH and treated with autologous ADSCs via portal vein injection. In each group, the rats were sacrificed at different time points postoperatively in order to evaluate the changes in liver function and to estimate the liver regenerative response. The expression of proliferating cell nuclear antigen (PCNA) labeling index in the liver was measured using immunohistochemistry. The expression levels of hepatocyte growth factor (HGF) mRNA were measured using reverse transcription polymerase chain reaction. The results showed that regeneration of the remaining liver following R-PH was significantly promoted by ADSC transplantation, as shown by a significant increase in liver to body weight ratio and the PCNA labeling index at $24 \mathrm{~h}$ post-hepatectomy. Additionally, ADSC transplantation markedly inhibited the elevation of serum levels of alanine aminotransferase, aspartate aminotransferase and total bilirubin, increased HGF content and also attenuated hepatic vacuolar degeneration $24 \mathrm{~h}$ postoperatively. Furthermore,
\end{abstract}

Correspondence to: Professor Yuliang Wang, Department of Clinical Laboratory Medicine, Tianjin First Central Hospital, Key Laboratory for Critical Care Medicine of the Ministry of Health, 24 Fukang Road, Nankai, Tianjin 300192, P.R. China

E-mail: wang_yu_1@163.com

Key words: repeat partial hepatectomy, liver regeneration, adipose tissue-derived mesenchymal stem cells the liver was found to almost fully recover from hepatocellular damage due to hepatectomy among the three groups at $168 \mathrm{~h}$ postoperatively. These results indicated that autologous ADSC transplantation enhanced the regenerative capacity of the remnant liver tissues in the early phase following R-PH.

\section{Introduction}

The most successful treatment option for hepatocellular carcinoma (HCC) is hepatectomy. However, the long-term survival rates following hepatectomy for HCC remain unsatisfactory due to the high rate of postoperative intrahepatic recurrence $(1,2)$. Despite this, in these patients, repeat hepatectomy has been reported to be a more effective therapeutic strategy for the treatment of recurrent hepatic tumors $(3,4)$. Even multiple bilobar metastases from colorectal cancer or carcinomas of other organs, which were previously considered a contraindication for hepatectomy, are now considered curable by planned two-staged hepatectomy under certain circumstances (5).

Following extensive hepatectomy, the regenerative capacity of the remnant liver is essential for patient survival (6). Accordingly, a novel therapeutic strategy is required for protection against liver dysfunction and for the enhancement of regenerative capacity. Fortunately, investigations into mesenchymal stem cells (MSCs) has offered a potential therapeutic tool in the field of liver regeneration (7). MSCs are an adult stem cells population with powerful proliferative and differentiation potential, which present an attractive tool for the establishment of successful stem cell-based therapy for liver diseases (8). Notably, several studies have focused on the role of MSCs in the liver regeneration process, and have reported that MSCs cam attenuate liver injuries and promote liver regeneration following partial hepatectomy (PH) (9-12). However, few studies have investigated the role of MSCs in liver regenerative following repeat partial hepatectomy (R-PH).

The aim of the present study was to investigate whether autologous adipose tissue-derived mesenchymal stem cell (ADSC) transplantation promoted the regeneration of the remaining liver tissues in a rat model of R-PH. 


\section{Materials and methods}

Animals. Male Wistar rats $(\mathrm{n}=60)$ aged 11 weeks and weighing 250-300 g were obtained from the Academy of Military Medical Science [Beijing, China; certificate no. SCXK (JUN) 2007-004]. These animals were maintained in a standard animal laboratory with free activity and free access to water and rodent chow. They were maintained in a temperature-controlled environment at $22-24^{\circ} \mathrm{C}$ with a $12-\mathrm{h}$ light-dark cycle. The rats were fasted for $12 \mathrm{~h}$ prior to surgery, and were provided with free access to $10 \%$ glucose water following surgery. All the surgical procedures were performed under sterile conditions, and all experiments were performed according to the National Institutes of Health Guide for Care and Use of Laboratory Animals (13) and were approved by the ethics committee of Tianjin First Central Hospital, Tianjin Medical University (Tianjin, China).

Establishment of the 70\% PH and R-PH models. For the introduction of $70 \% \mathrm{PH}$, the 60 rats were anesthetized with isoflurane inhalation (Lunan Pharmaceutical Co., Ltd., Shandong, China) via an isoflurane vaporizer (Matrx VMR; Midmark corporation, Dayton, OH, USA), and 70\% of the liver of each rat, comprising the left lateral and median lobes, was excised, using the technique described by Saito et al (14). Suturing of the peritoneum and skin were performed independently. The remaining $30 \%$ of the liver started to grow for 7 days, following which R-PH was performed, in which 40 of 60 rats were anesthetized and the right lateral lobe was ligated and excised.

Isolation and culture of autologous ADSCs. To obtain adequate cells and avoid the requirement for a long duration following establishment of the PH model, the autologous ADSCs were isolated and expanded 2 weeks prior to surgery. Adipose tissue cells were isolated from all 60 rats using a described previously method (15). Briefly, the rats were anesthetized via inhalational isoflurane. The hemi-inguinal fat pads were carefully excised and minced into pieces of $\sim 1 \mathrm{~mm}^{3}$. The adipose tissue was digested in collagenase type I solution (Sigma-Aldrich, St. Louis, MO, USA) for $60 \mathrm{~min}$ at $37^{\circ} \mathrm{C}$ with constant agitation (100 rpm). The stromal cells were separated from the floating adipocytes by centrifugation at $200 \mathrm{~g}$ for $5 \mathrm{~min}$ at room temperature. The cells released were then resuspended in Dulbecco's modified Eagle's medium (DMEM)/F12 medium (Gibco; Thermo Fisher Scientific, Inc., Waltham, MA, USA), and then sieved through $70 \mu \mathrm{m}$ mesh (BD Biosciences, Franklin Lakes, NJ, USA). The resulting ADSCs were cultivated in DMEM/F12 medium containing 10\% FBS (Gibco; Thermo Fisher Scientific, Inc.). Following in vitro culture for 14 days at $37^{\circ} \mathrm{C}, 5 \% \mathrm{CO}_{2}$ and $95 \%$ humidity, a sufficient number of ADSCs were obtained for the autologous transplantation. The ADSCs $\left(3 \times 10^{6}\right)$ from each experimental rat were cryopreserved in liquid nitrogen (Air Products and Chemicals (Tianjin) Co., Ltd., Tianjin, China) with cell name marked on tube prior to injection.

Cell surface antigen profile of ADSCs. The expression levels of cell surface antigen were evaluated using flow cytometry. When cultures reach $>80 \%$ confluency at $37^{\circ} \mathrm{C}, 5 \% \mathrm{CO}_{2}$ and $95 \%$ humidity, adherent cells were removed from the tissue culture polystyrene flasks via trypsinization (Invitrogen; Thermo Fisher Scientific, Inc.) and washed twice with DMEM/F12. All cells were incubated with fluorescein isothiocyanate-conjugated mouse anti-rat monoclonal antibodies against rat CD45 (cat. no. 554877), CD73 (cat. no. 551123), CD90 (cat. no. 554894) all obtained from BD Biosciences), CD34 (cat. no. sc-7324; Santa Cruz Biotechnology, Inc., Santa Cruz, CA, USA) and CD105 (cat. no. ab11414; Abcam, Cambridge, MA, USA) for $40 \mathrm{~min}$ at room temperature at a dilution of 1:50. Following antibody incubation, data were acquired using a FACSCalibur flow cytometer (BD Biosciences) and analyzed using CellQuest 6.0 software (BD Biosciences).

Multidifferentiation ability of ADSCs. The differentiation of the cells into osteogenic and adipogenic lineages, and subsequent detection were performed using established methodologies (15). Briefly, the ADSCs were seeded in medium at $2 \times 10^{4}$ cells $/ \mathrm{cm}^{2}$ in six-well tissue culture plates. When the cells reached $100 \%$ confluency, DMEM/F12 was subsequently replaced with osteogenic inducer medium containing $100 \mathrm{nmol} / 1$ dexamethasone (Sigma-Aldrich), $10 \mathrm{mmol} / \mathrm{l} \beta$-sodium glycerophosphate (Sigma-Aldrich) and $50 \mu \mathrm{g} / \mathrm{ml}$ vitamin C (Sigma-Aldrich), or adipogenic inducer medium containing $1 \mu \mathrm{mol} / 1$ dexamethasone, $0.5 \mathrm{mmol} / 1$ 3-isobutyl-1-methylxanthine (Sigma-Aldrich), $5 \mathrm{mg} / \mathrm{l}$ insulin (Sigma-Aldrich) and $100 \mu \mathrm{mol} / \mathrm{l}$ indomethacin (Sigma-Aldrich), in DMEM/F12. Cells were maintained at $37^{\circ} \mathrm{C}$ in a $5 \% \mathrm{CO}_{2}$ incubator and the medium was changed every 3 days. Following a 14 day induction period, the cells were assayed for mineral content by Von Kossa staining (Shanghai Genmed Gene Pharmaceutical Technology Co., Ltd., Shanghai, China) and for lipid accumulation using Oil Red O staining (Sigma-Aldrich).

Experimental groups, cell transplantation and sample collection. The rats were divided into the following three groups: PH $(n=20)$; R-PH $(n=20)$, subjected to a R-PH and treated with saline by portal vein injection; and R-PH/ADSC group $(n=20)$, subjected to R-PH and treated with autologous ADSCs $\left(2 \times 10^{6}\right.$ cells/rat) by portal vein injection. Subsequent to these procedures, five animals in each group were sacrificed using anesthesia, as described above, at 24, 72 and $168 \mathrm{~h}$ following hepatectomy, respectively. Blood samples $(2 \mathrm{ml})$ were collected by puncturing the vena cava, and the residual liver lobes were then rapidly excised and weighed. The livers were fixed in formalin (Tianjin Kemiou Chemical Reagent Co., Ltd., Tianjin, China) overnight, prior to processing and embedding in paraffin wax (Tianjin Kemiou Chemical Reagent Co., Ltd.). Sections (5- $\mu \mathrm{m}$ thick) were deparaffinized and fixed. The sections were stained with hematoxylin and eosin (H\&E; Sigma-Aldrich) and observed using a Nikon Ni-U fluorescence microscope (Nikon Corporation, Tokyo, Japan) Additional samples were stored in liquid nitrogen.

Liver mass and function recovery. For each time point, the total body weight of each of the fasted rats were weighed prior to sacrifice. The regenerating ratio of the liver following hepatectomy was calculated as the liver wet weight to body weight ratio (LBR), rather than the weight of the remnant lobes alone. The $2 \mathrm{ml}$ blood samples were centrifuged at $4,000 \mathrm{x} \mathrm{g}$ 
for $10 \mathrm{~min}$ at room temperature prior to serum collection. The serum concentrations of alanine aminotransferase (ALT), aspartate aminotransferase (AST) and total bilirubin (TBIL) were measured using an automatic biochemical analyzer (Hitachi 7600; Hitachi, Ltd., Tokyo, Japan) 24, 72, and 168 h postoperatively in the R-PH/ADSC, R-PH and PH groups.

Proliferating cell nuclear antigen (PCNA)-labeling index. The expression level of PCNA, determined by immunohistochemistry, correlates with the degree of cell proliferation (16). Briefly, following fixation with formalin and paraffin embedding, the liver tissue sections were incubated with rabbit anti-rat polyclonal antibody against PCNA (cat. no. GTX100539; 1:500 dilution; Genetex Inc. Irvine, CA, USA) at $4^{\circ} \mathrm{C}$ overnight, and subsequently with a 3',3-diaminobenzidine kit (Beyotime Institute of Biotechnology, Haimen, China). The proliferation index of the PCNA-stained cells was measured by counting the number of positive nuclei of hepatocytes under Ni-U fluorescence microscope, with data expressed as the percentage of PCNA-stained hepatocytes of the total number of hepatocytes.

mRNA expression of hepatocyte growth factor (HGF). The mRNA levels of HGF in liver tissue were measured using reverse transcription-quantitative polymerase chain reaction (RT-qPCR) analysis. Total RNA was extracted from the frozen remnant lobe samples using TRIzol reagent (Invitrogen; Thermo Fisher Scientific, Inc.), and then subjected to RT using a High Capacity cDNA Reverse Transcription kit (Applied Biosystems; Thermo Fisher Scientific, Inc.). qPCR was performed using an ABI 7500 Sequence Detection System (Applied Biosystems; Thermo Fisher Scientific, Inc.) using $1 \mu \mathrm{l}$ cDNA template and 1X SYBR-Green I (Takara Bio, Inc., Tokyo, Japan) in a $25 \mu \mathrm{l}$ reaction mixture (Takara Bio, Inc.; $10 \mathrm{mM}$ Tris- $\mathrm{HCl}, 50 \mathrm{mM}$ $\mathrm{KCl}, 1.5 \mathrm{mM} \mathrm{MgCl}_{2}, 200 \mu \mathrm{M}$ dNTP mix, $0.2 \mu \mathrm{M}$ of each primer and 1 unit of Taq DNA polymerase). Primer Premier V5.0 software was used to design the primers, according to HGF gene sequences (GenBank; www.ncbi.nlm.nih.gov/genbank). Primers were synthesized by Integrated DNA Technologies (Coralville, IA, USA). The primer sequences were as follows: HGF, sense 5'-ACAGCT TTT TGCCTTCGAGCTA-3' and anti-sense 5'-CATCAAAGCCCTTGTCGGGATA-3'; $\beta$-actin, sense, 5'-ATATCGCTGCGCTCGTCGTC-3' and anti-sense 5'-TCTTGCTCTGGGCCTCGTC-3'. The conditions for each qPCR reaction were as follows: $30 \mathrm{sec}$ at $95^{\circ} \mathrm{C}$, followed by 40 cycles of denaturation for $5 \mathrm{sec}$ at $95^{\circ} \mathrm{C}$, annealing for $30 \mathrm{sec}$ at $58^{\circ} \mathrm{C}$ and extension for $30 \mathrm{sec}$ at $72^{\circ} \mathrm{C}$. The level of expression was calculated using the $2^{-\Delta \mathrm{Cq}}$ method, in which $\Delta \mathrm{Cq}$ was calculated as $\mathrm{Cq}$ of target molecule - Cq of $\beta$-actin (17).

Statistical analysis. Data are expressed as the mean \pm standard deviation. Differences in parameters were analyzed using one-way analysis of variance. Statistical analyses were performed using SPSS 16.0 software (SPSS, Inc., Chicago, IL, USA). $\mathrm{P}<0.05$ was considered to indicate a statistically significant difference.

\section{Results}

Characterization of rat ADSCs. The ADSCs were verified by analyzing the expression surface markers and multipotent

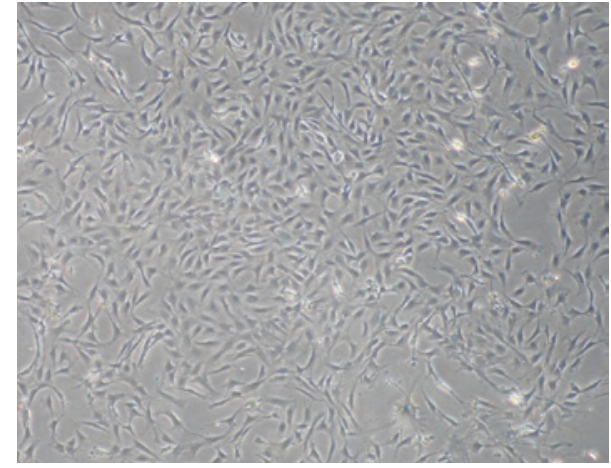

Figure 1. Representative image showing the morphology of rat ADSCs in normal male Wistar rats. The ADSCs at passage three exhibited a spindle-shaped fibroblastic morphology (magnification, x100). ADSCs, adipose tissue-derived mesenchymal stem cells.

differentiation of the cells. At passage three, the cultured ADSCs exhibited a fibroblast-like morphology (Fig. 1). The data showed that the ADSCs were positive for CD90, CD105 and CD73, but were negative for CD34 and CD45 when analyzed using flow cytometric analyses (Fig. 2). In addition, the ADSCs at passage three exhibited potential for osteogenic and adipogenic differentiation following culture in osteogenic and adipogenic growth media. Positive ALP and oil red $\mathrm{O}$ staining confirmed the cells as osteogenic and adipogenic, respectively (Fig. 3).

Effect of ADSCs on remnant liver regeneration. The outcomes in the 60 rats subjected to $\mathrm{PH}$ were examined in the present study. The LBR at 24 and $72 \mathrm{~h}$ post-hepatectomy was significant decreased in the R-PH group, compared with that in the $\mathrm{PH}$ group $(\mathrm{P}<0.01$; Fig. 4). However, the LBR in the R-PH rats that received ADSC transplantation, was significantly higher, compared with that in the R-PH rats $72 \mathrm{~h}$ postoperatively $(\mathrm{P}<0.05)$. Furthermore, the LBR increased steadily in the three groups, and no further differences among the groups were observed $168 \mathrm{~h}$ postoperatively (Fig. 4).

Effects of ADSC transplantation on liver histopathology. At $\sim 24 \mathrm{~h}$ post-hepatectomy, the sections of the remnant lobes were stained with H\&E and examined under a light microscope. The histopathological analysis revealed that no liver cell inflammation or necrosis was present in any of the samples. In the PH group, the arrangement of hepatocytes was deranged, with microvesicular fatty degeneration observed in the cytoplasm and mild dilatation of the sinusoids (Fig. 5A). The remnant lobe in the R-PH group exhibited prevalent vacuolization degeneration in the hepatocytes, and dilatation of the sinusoids was more marked, compared with that in the PH group (Fig. 5B). Similarly, in the R-PH/ADSC group, vacuolization and sinusoidal dilatation was similar, but to a lesser extent, compared with the R-PH group (Fig. 5C).

Effects of ADSC transplantation on liver function following $70 \% \mathrm{PH}$. In the R-PH/ADSC group, the levels of ALT and TBIL were substantially lower $24 \mathrm{~h}$ following hepatectomy, compared with those in the R-PH group, whereas the levels of AST were significantly lower 24 and $72 \mathrm{~h}$ postoperatively, compared with those in the R-PH group. Although a significant 

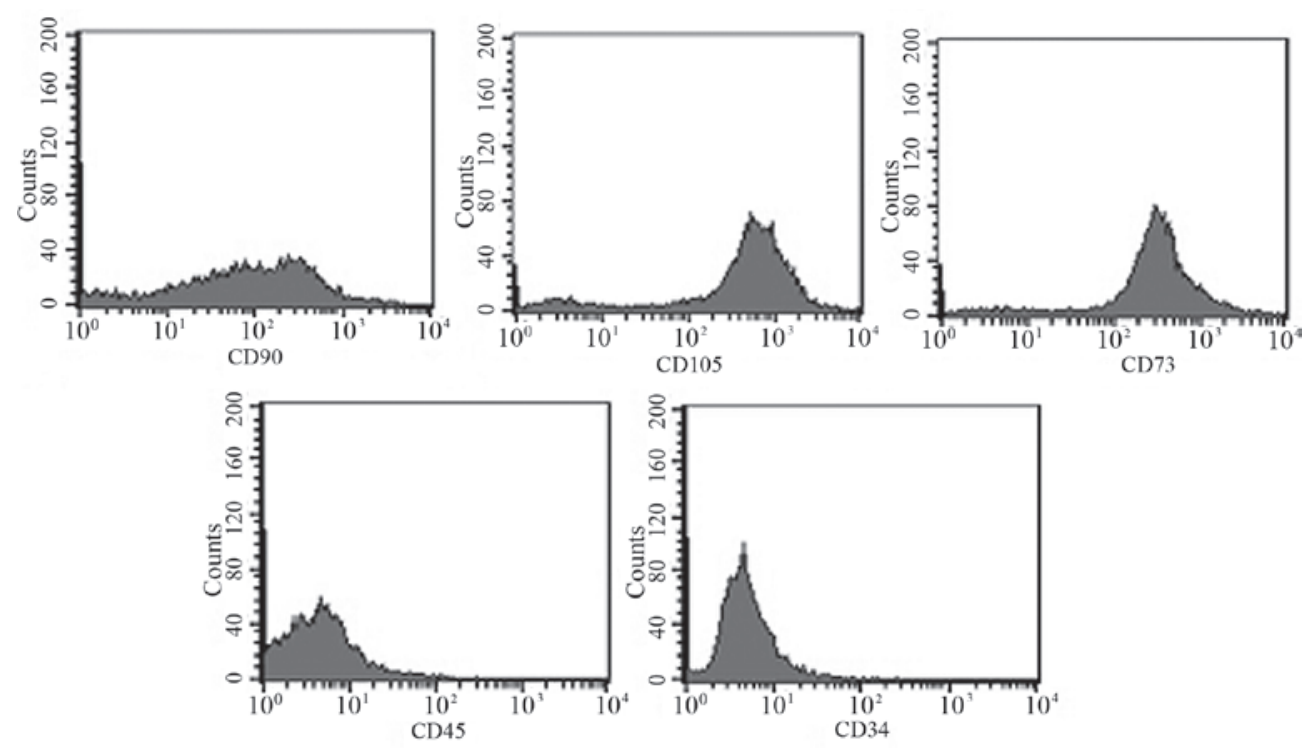

Figure 2. Phenotypic characterization of ADSCs. The ADSCs from passage the were stained with the indicated antibodies and analyzed using flow cytometry. This indicated that ADSCs are positive for CD90, CD105 and CD73, however, they are negative for CD45 and CD34. ADSCs, adipose tissue-derived mesenchymal stem cells; $\mathrm{CD}$, cluster of differentiation.
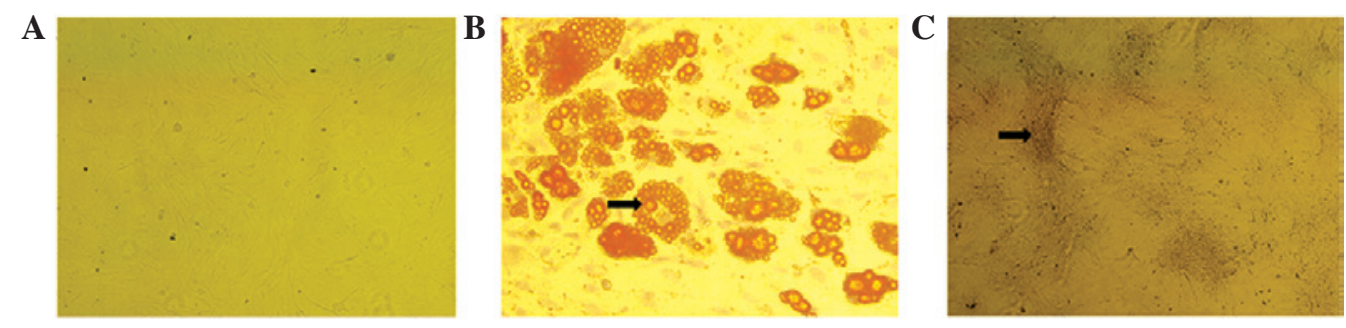

Figure 3. Multipotent differentiation of ADSCs in vitro. (A) Parental ADSCs; (B) adipocyte differentiation, the arrows indicate red lipid droplets; (C) osteoblast differentiation, the arrows indicate black calcium deposition. ADSCs, adipose tissue-derived mesenchymal stem cells. Magnification, x100.

protective effect was observed in the rats transplanted with ADSCs, the liver function remained significantly higher in the R-PH group, compared with that in the $\mathrm{PH}$ group $24 \mathrm{~h}$ postoperatively. At $72 \mathrm{~h}$ postoperatively, the serum levels of ALT and TBIL in the three groups were similar. Furthermore, the liver function gradually decreased to the basal level in the three groups at $168 \mathrm{~h}$ post-hepatectomy (Fig. 6).

Effect of ADSCs on the PCNA-labeling index. To evaluate whether ADSC transplantation enhanced the proliferation of hepatocytes in remnant lobes, the expression levels of PCNA were assessed. Although the number of PCNA-positive hepatocytes was significantly reduced in the R-PH rats transplanted with ADSCs, compared with the $\mathrm{PH}$ rats $(\mathrm{P}<0.01$; Fig. 7), the R-PH rats transplanted with ADSCs exhibited a significant increase in the number of PCNA-positive hepatocytes at $24 \mathrm{~h}$ postoperatively, compared with the R-PH rats, indicating that ADSCs exerted hepatoprotective effects by promoting liver regeneration in the early phase. No statistically significant differences in the PCNA-labeling index were observed among three groups at 72 and $168 \mathrm{~h}(\mathrm{P}>0.05$; Fig. 7).

Effect of ADSCs on the mRNA levels of HGF. The mRNA levels of HGF peaked within $24 \mathrm{~h}$ in the PH group and R-PH/ADSC

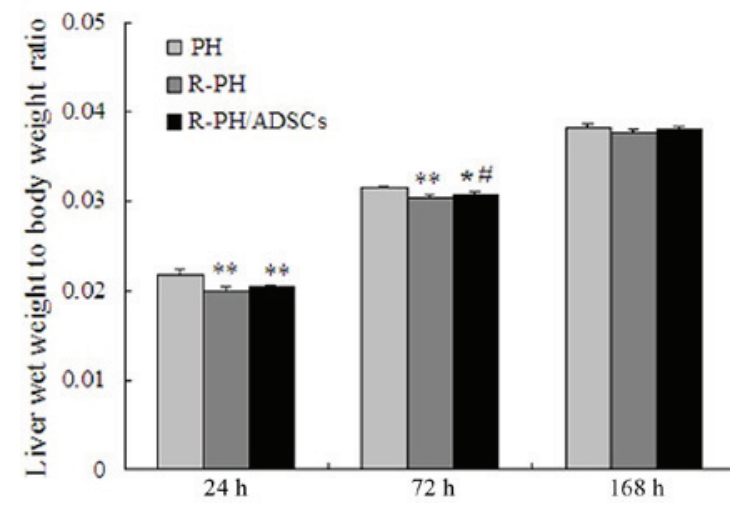

Figure 4. Effect of ADSCs on the LBR at 24, 72 and $168 \mathrm{~h}$ postoperatively. Data are expressed as the mean \pm standard deviation of the results from five animals. ${ }^{*} \mathrm{P}<0.05$ and ${ }^{* *} \mathrm{P}<0.01$, vs. $\mathrm{PH}$ group; ${ }^{*} \mathrm{P}<0.05$, vs. $\mathrm{R}-\mathrm{PH}$ group. ADPCs, adipose tissue-derived mesenchymal stem cells; LBR, liver wet weight to body weight ratio; $\mathrm{PH}$, partial hepatectomy; R-PH, repeat $\mathrm{PH}$.

group, and then gradually decreased after $72 \mathrm{~h}$. However, the mRNA levels of HGF in the R-PH group decreased significantly after $24 \mathrm{~h}$, and peaked at $72 \mathrm{~h}$, compared with $\mathrm{PH}$ and $\mathrm{R}-\mathrm{PH} / \mathrm{ADSC}$ groups, indicating that hepatic regeneration following R-PH was inhibited. The postoperative course of the mRNA levels of HGF are shown in Fig. 8. 


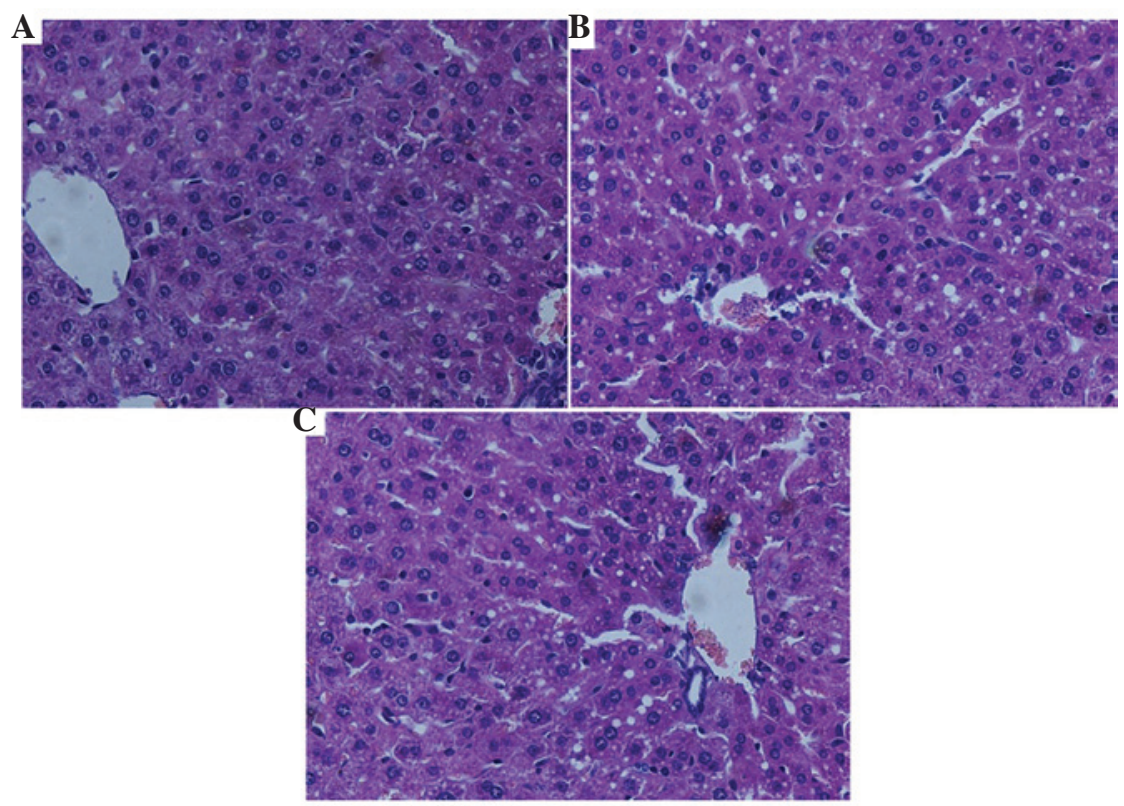

Figure 5. Effects of ADSC transplantation on liver histopathology at $24 \mathrm{~h}$ postoperatively. (A) In the PH group, derangement of liver cells and mild sinusoidal dilatation were evident, and small vacuoles were present in the hepatocytes. (B) In the R-PH group, vacuolization was prevalent in the hepatocytes and sinusoidal dilatation was evident. (C) In the R-PH/ADSC group, vacuolization degeneration and sinusoidal dilatation were observed, but to a lesser extent, compared with the PH and R-PH groups. (Magnification, x200). ADSCs, adipose tissue-derived mesenchymal stem cells; PH, partial hepatectomy; R-PH, repeat $\mathrm{PH}$.
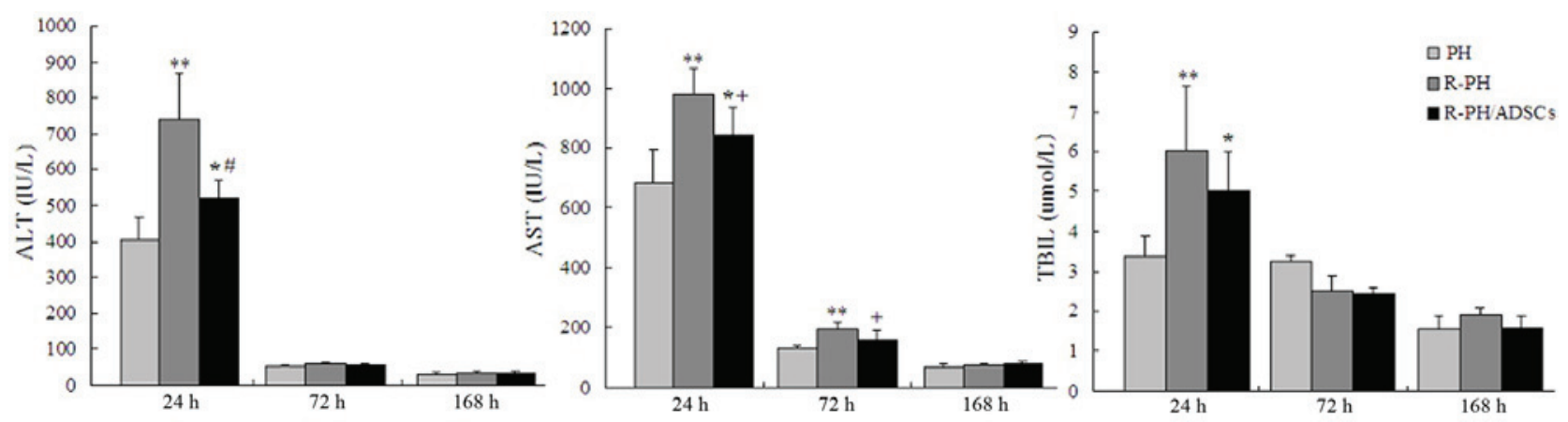

Figure 6. Effects of ADSC transplantation on liver function at 24, 72, and $168 \mathrm{~h}$ postoperatively. Levels of ALT, AST and TBIL were analyzed as indicators of liver function. Data are expressed as the mean \pm standard deviation of the results from five animals. ${ }^{*} \mathrm{P}<0.05$ and ${ }^{* *} \mathrm{P}<0.01$, vs. $\mathrm{PH}$ group; ${ }^{+} \mathrm{P}<0.05$, vs. $\mathrm{R}-\mathrm{PH}$ group; " $\mathrm{P}<0.01$, vs. R-PH group. ADSC, adipose tissue-derived mesenchymal stem cell; PH, partial hepatectomy; R-PH, repeat PH; ALT, alanine aminotransferase; AST, aspartate aminotransferase; TBIL, total bilirubin.

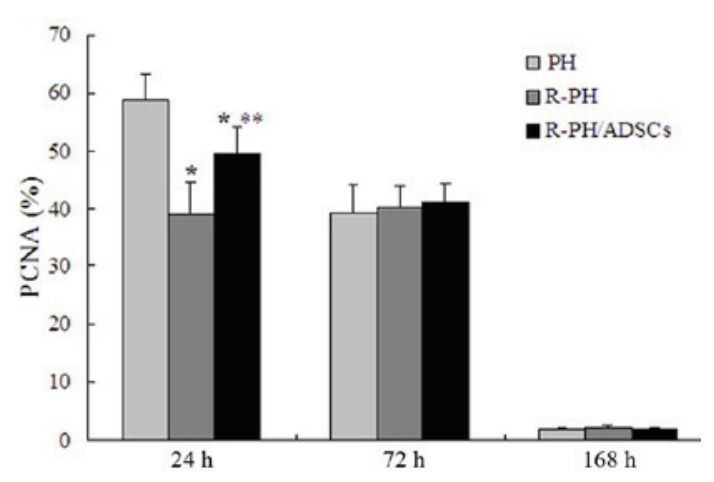

Figure 7. Effect of ADSC transplantation on the PCNA-labeling index at 24,72 , and $168 \mathrm{~h}$ postoperatively. Data are expressed as the mean \pm standard deviation of the results from five animals. " $\mathrm{P}<0.01$, vs. $\mathrm{PH}$ group; ${ }^{* *} \mathrm{P}<0.01$, vs. R-PH group. ADSC, adipose tissue-derived mesenchymal stem cell; PCNA, proliferating cell nuclear antigen; $\mathrm{PH}$, partial hepatectomy; $\mathrm{R}-\mathrm{PH}$, repeat $\mathrm{PH}$.

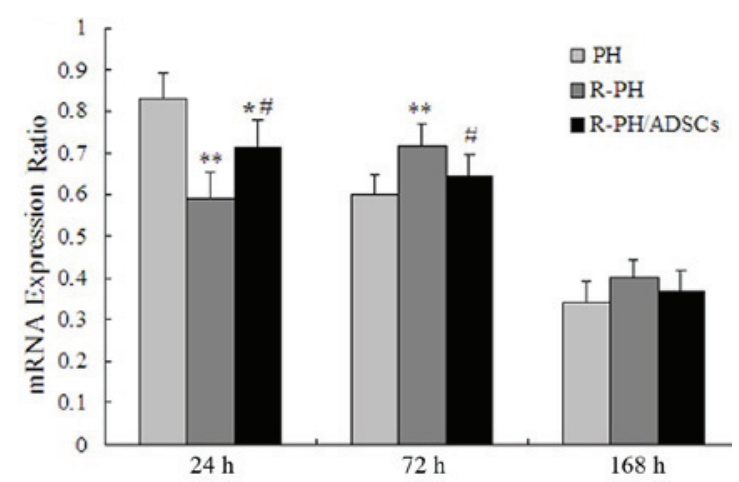

Figure 8. Effect of ADSC transplantation on the mRNA expression levels of hepatocyte growth factor at 24,72 , and $168 \mathrm{~h}$ postoperatively. Data are expressed as the mean \pm standard deviation of the results from five animals. ${ }^{*} \mathrm{P}<0.05$ and ${ }^{* *} \mathrm{P}<0.01$, vs. $\mathrm{PH}$ group; ${ }^{*} \mathrm{P}<0.05$, vs. $\mathrm{R}-\mathrm{PH}$ group. ADSC, adipose tissue-derived mesenchymal stem cell; $\mathrm{PH}$, partial hepatectomy; R-PH, repeat $\mathrm{PH}$. 


\section{Discussion}

HCC is the fifth most common type of cancer worldwide, and has a mortality rate of 500,000 globally every year (18). The long-term survival rates of patients following hepatectomy remain unsatisfactory due to the high incidence of recurrence. Clinically, repeat hepatectomy can be performed safely, and is associated with long-term survival rates in a subset of patients with recurrent HCC (19). In order to avoid liver dysfunction, also termed small-for-size syndrome, the regenerative capacity of the remnant liver is essential for patient survival (20). ADSCs have been considered as an attractive and readily available type of adult MSC, and are becoming increasingly popular for use in regenerative cell therapy as they are readily accessible through minimally invasive methods and can be used for autologous transplantation (21). In the present study, autologous ADSC transplantation significantly enhanced liver regenerative capacity following R-PH in rats, as indicated by an increased LBR and PCNA-label index. In addition, autologous ADSC transplantation alleviated R-PH-induced liver injury, as evidenced by inhibition in the elevated serum levels of ALT, AST and TBIL, and the improvement of pathological changes.

As a initial step in the present study, the LBR was assessed as the liver growth kinetics of regeneration postoperatively, which is the most direct index for evaluating liver regeneration (22). The results showed that regeneration in the liver tissue began from the first day following PH. The mean LBR increased progressively among three groups postoperatively. However, the LBR decreased significantly in the R-PH group at 24 and $72 \mathrm{~h}$ postoperatively, compared with the PH group, indicating that the hepatic regeneration response following R-PH was significantly attenuated at the initial stage, which is in agreement with previous data (23). Furthermore, no statistically significant differences were observed between $\mathrm{PH}$ and $\mathrm{R}-\mathrm{PH}$ at $168 \mathrm{~h}$ postoperatively, indicating that 7 days was a sufficient period of time for these animals to recover from the surgical stresses of a R-PH. Of note, the LBR in the R-PH rats which received ADSC transplantation was significantly higher, compared with that in the R-PH rats, indicating that the ADSCs promoted the rapid regeneration of hepatocyte numbers in the initial stage (24). Consistent with the above results, the administration of conventionally cultured autologous ADSCs in the present study also resulted in proliferation of remnant hepatocytes $24 \mathrm{~h}$ following R-PH in the rats, which was reflected by the elevated expression of PCNA-positive cells, suggesting that the beneficial effects of ADSCs on hepatic regeneration was more active at the cellular level than following R-PH alone. In addition, the expression levels of PCNA-positive cells were gradually decreased at 72 and $168 \mathrm{~h}$ among three groups, which suggested that hepatocyte proliferation occurred at a faster rate initially, and more slowly during progression.

The present study also demonstrated that the duration of recovery of liver volume closely coincided with that of hepatocellular damage. The present study showed that the rats subjected to ADSC transplantation via the portal vein following R-PH showed increased improvement of liver function at $24 \mathrm{~h}$, compared with the corresponding liver function in the R-PH group. However, the liver function in the R-PH/ADSC group remained inferior to that in the $\mathrm{PH}$ group, as demonstrated by the serum levels of ALT, AST and TBIL. Therefore, the improvement of liver function by ADSC transplantation was partially contributed to through the enhancement of hepatocyte proliferation. This is consistent with the histopathological finding, in which the R-PH rats exhibited derangement of hepatocyte structure, extensive lipid vacuolization of hepatocytes and sinusoid dilatation in the remnant lobe at $24 \mathrm{~h}$ postoperatively. However, vacuolization and sinusoidal dilatation, was apparent to a lesser extent in the R-PH/ADSC group, suggesting that ADSC transplantation may attenuate liver injury and lead to the earlier reconstitution of residual liver tissue.

The concept of stem cell transplantation exerting a paracrine proliferative effect on endogenous hepatocytes is gaining support. The present study examined the expression levels of HGF, which has been demonstrated to be the most potent stimulator of hepatocyte growth and DNA synthesis in vitro, as well as one of the key regulators of liver regeneration following $\mathrm{PH}$ or hepatic injury $(25,26)$. In the present study, the mRNA levels of HGF peaked within $24 \mathrm{~h}$ in the $\mathrm{PH}$ and R-PH/ADSC groups, and then gradually decreased after $72 \mathrm{~h}$. Of note, the mRNA levels of HGF in the R-PH group were significantly decreased after $24 \mathrm{~h}$, following which they increased to peak at $72 \mathrm{~h}$ postoperatively, compared with the PH and R-PH/ADSC groups. This is in agreement with a report by Saito et al, who observed that R-PH rats exhibit inhibited hepatic regeneration during the early postoperative phase due to the depressed expression of HGF (14). It has been shown that MSCs synthesize a wide variety of growth factors and cytokines, exerting a paracrine effect on local cellular dynamics (27). Therefore, the results of the present study support the viewpoint that the predominant advantage provided by ADSCs transplanted via the portal vein is the acceleration in the production of HGF in the early period through paracrine effects, supporting this method as an effective treatment strategy in liver regeneration. However, the follow-up period in the present study was limited, therefore, it is not possible to exclude that, in a longer period of observation, ADSCs as an efficient alternative source can undergo hepatogenic differentiation. However, in previous experiments involving the administration of bone marrow MSCs in CCl4-treated mice, for 4 weeks, only a small percentage of the MSCs underwent hepatocyte-like differentiation (28).

In conclusion, the results of the present study suggested that the transplantation of autologous ADSCs reduced liver injury and promoted hepatocyte proliferation, particularly during the first $24 \mathrm{~h}$ following R-PH. The upregulation of HGF may mediate the therapeutic effects of these transplanted ADSCs.

\section{Acknowledgements}

The present study was supported by the National Natural Science Foundation of China (grant nos. 81470982 and 81402322), the State-funded Construction Projects-Key Specialized Subject of Clinical Laboratory Medicine (grant no. 2013-544), Tianjin Research Program of Application Foundation and Advanced Technology (grant no. 13JCYBJC23000) and the Technology Foundation of Tianjin Municipal Health Bureau (grant no. 2014KZ028). 


\section{References}

1. Llovet JM, Burroughs A and Bruix J: Hepatocellular carcinoma. Lancet 362: 1907-1917, 2003.

2. Forner A, Llovet JM and Bruix J: Hepatocellular carcinoma. Lancet 379: 1245-1255, 2012.

3. Kubo S, Takemura S, Uenishi T, Yamamoto T, Ohba K, Ogawa M, Hai S, Ichikawa T, Kodai S, Shinkawa $\mathrm{H}$ and Tanaka H: Second hepatic resection for recurrent hepatocellular carcinoma in patients with chronic hepatitis C. World J Surg 32: 632-638, 2008

4. Zhou Y, Sui C, Li B, Yin Z, Tan Y, Yang J and Liu Z: Repeat hepatectomy for recurrent hepatocellular carcinoma: A local experience and a systematic review. World J Surg Oncol 8: 55, 2010.

5. Wicherts DA, de Haas RJ, Salloum C, Andreani P, Pascal G, Sotirov D, Adam R, Castaing D and Azoulay D: Repeat hepatectomy for recurrent colorectal metastases. Br J Surg 100: 808-818, 2013

6. Tralhão JG, Abrantes AM, Hoti E, Oliveiros B, Cardoso D Faitot F, Carvalho C, Botelho MF and Castro-Sousa F: Hepatectomy and liver regeneration: From experimental research to clinical application. ANZ J Surg 84: 665-671, 2014.

7. Drosos I and Kolios G: Stem cells in liver regeneration and their potential clinical applications. Stem Cell Rev 9: 668-684, 2013.

8. Du Z, Wei C, Cheng K, Han B, Yan J, Zhang M, Peng C and Liu Y: Mesenchymal stem cell-conditioned medium reduces liver injury and enhances regeneration in reduced-size rat liver transplantation. J Surg Res 183: 907-915, 2013.

9. Li DL, He XH, Zhang SA, Fang J, Chen FS and Fan JJ: Bone marrow-derived mesenchymal stem cells promote hepatic regeneration after partial hepatectomy in rats. Pathobiology 80 228-234, 2013

10. Li T, Zhu J, Ma K, Liu N, Feng K, Li X, Wang S and Bie P: Autologous bone marrow-derived mesenchymal stem cell transplantation promotes liver regeneration after portal vein embolization in cirrhotic rats. J Surg Res 184: 1161-1173, 2013.

11. Koellensperger E, Niesen W, Kolbenschlag J, Gramley F, Germann $\mathrm{G}$ and Leimer U: Human adipose tissue derived stem cells promote liver regeneration in a rat model of toxic injury. Stem Cells Int 2013: 534263, 2013.

12. Kaibori M, Adachi Y, Shimo T, Ishizaki M, Matsui K, Tanaka Y, Ohishi M, Araki Y, Tokuhara K, Okumura T, et al: Bone marrow cells enhance liver regeneration after massive hepatectomy in mice. Dig Dis Sci 59: 1484-1489, 2014.

13. Clark JD, Gebhart GF, Gonder JC, Keeling ME and Kohn DF: The 1996 Guide for the Care and Use of Laboratory Animals ILAR J 38: 41-48, 1997

14. Saito S, Togo S, Morioka D, Matsuo K, Yoshimoto N, Nagano Y, Tanaka K, Kubota T, Nagashima Y and Shimada H: A rat model of a repeat 70\% major hepatectomy. J Surg Res 134: 322-326, 2006.
15. Wang YL, Li G, Zou XF, Chen XB, Liu T and Shen ZY: Effect of autologous adipose-derived stem cells in renal cold ischemia and reperfusion injury. Transplant Proc 45: 3198-3202, 2013.

16. Ding L, Yang Y, Qu Y, Yang T, Wang K, Liu W and Xia W: Bile acid promotes liver regeneration via farnesoid $X$ receptor signaling pathways in rats. Mol Med Rep 11: 4431-4437, 2015.

17. Wang Y, Wang Y, Mu H, Liu T, Chen XB and Shen ZY: Enhanced specific antitumor immunity of dendritic cells transfected with glypican 3 gene and co-cultured with cytokine-induced killer cells against hepatocellular carcinoma cells. Mol Med Rep 11: 3361-3367, 2015 .

18. Jemal A, Bray F, Center MM, Ferlay J, Ward E and Forman D: Global cancer statistics. CA Cancer J Clin 61: 69-90, 2011.

19. Chan DL, Morris DL and Chua TC: Clinical efficacy and predictors of outcomes of repeat hepatectomy for recurrent hepatocellular carcinoma-a systematic review. Surg Oncol 22: e23-30, 2013

20. Serenari M, Cescon M, Cucchetti A and Pinna AD: Liver function impairment in liver transplantation and after extended hepatectomy. World J Gastroenterol 19: 7922-7929, 2013.

21. Ishikawa T, Banas A, Hagiwara K, Iwaguro H and Ochiya T: Stem cells for hepatic regeneration: The role of adipose tissue derived mesenchymal stem cells. Curr Stem Cell Res Ther 5: 182-189, 2010.

22. Dusabineza AC, Van Hul NK, Abarca-Quinones J, Starkel P, Najimi M and Leclercq IA: Participation of liver progenitor cells in liver regeneration: Lack of evidence in the $\mathrm{AAF} / \mathrm{PH}$ rat model. Lab Invest 92: 72-81, 2012.

23. Aoki T, Murakami M, Niiya T, Murai N, Shimizu Y, Kato H and Kusano M: Capacity of hepatic regeneration following a second partial hepatectomy in rats. Hepatol Res 21: 228-241, 2001.

24. Salomone F, Barbagallo I, Puzzo L, Piazza C and Li Volti G: Efficacy of adipose tissue-mesenchymal stem cell transplantation in rats with acetaminophen liver injury. Stem Cell Res 11: 1037-1044, 2013

25. Sun J, Yuan Y, Qin H, Ying C, Liu W, Zhang J, He Y and Liu Z Serum from hepatectomized rats induces the differentiation of adipose tissue mesenchymal stem cells into hepatocyte-like cells and upregulates the expression of hepatocyte growth factor and interleukin-6 in vitro. Int J Mol Med 31: 667-675, 2013.

26. Nejak-Bowen K, Orr A, Bowen WC Jr and Michalopoulos GK: Conditional genetic elimination of hepatocyte growth factor in mice compromises liver regeneration after partial hepatectomy. PLoS One 8: e59836, 2013.

27. Nikoozad Z, Ghorbanian MT and Rezaei A: Comparison of the liver function and hepatic specific genes expression in cultured mesenchymalstem cells and hepatocytes. Iran J Basic Med Sci 17: 27-33, 2014.

28. Li Q, Zhou X, Shi Y, Li J, Zheng L, Cui L, Zhang J, Wang L, Han Z, Han Y and Fan D: In vivo tracking and comparison of the therapeutic effects of MSCs and HSCs for liver injury. PLoS One 8: e62363, 2013. 